\title{
Local emergence of thermal correlations in an isolated quantum many-body system
}

\author{
T. Langen ${ }^{\star}$, R. Geiger, M. Kuhnert, B. Rauer and J. Schmiedmayer ${ }^{\star}$
}

\begin{abstract}
Understanding the dynamics of isolated quantum manybody systems is a central open problem at the intersection between statistical physics and quantum physics. Despite important theoretical effort ${ }^{1}$, no generic framework exists yet to understand when and how an isolated quantum system relaxes to a steady state. Regarding the question of how, it has been conjectured ${ }^{2,3}$ that equilibration must occur on a local scale in systems where correlations between distant points can establish only at a finite speed. Here, we provide the first experimental observation of this local equilibration hypothesis. In our experiment, we quench a one-dimensional Bose gas by coherently splitting it into two parts. By monitoring the phase coherence between the two parts we observe that the thermal correlations of a prethermalized state $e^{4,5}$ emerge locally in their final form and propagate through the system in a light-cone-like evolution. Our results underline the close link between the propagation of correlations $s^{2,3,6,7}$ and relaxation processes in quantum many-body systems.
\end{abstract}

It has been theoretically suggested that relaxation in generic isolated quantum many-body systems proceeds through the dephasing of the quantum states populated at the onset of the non-equilibrium evolution ${ }^{8,9}$. It is generally believed that this dynamically leads to relaxed states that can be well described either by the usual thermodynamical ensembles or by generalized Gibbs ensembles that take into account dynamical constraints ${ }^{10}$. However, it remains an open question how these relaxed states form dynamically, and in particular, whether they emerge gradually on a global scale, or appear locally and then spread in space and time ${ }^{3}$.

Ultracold atomic gases offer an ideal test bed to explore such quantum dynamics. Their almost perfect isolation from the environment and the many available methods to probe their quantum states make it possible to reveal the dynamical evolution of a many-body system at a very detailed level ${ }^{4,7,11-16}$.

In our experiment, a phase-fluctuating ultracold onedimensional (1D) Bose gas ${ }^{17}$ is split coherently ${ }^{18}$. The splitting creates a non-equilibrium state consisting of two gases with almost identical phase profiles. Interactions in the many-body system drive the relaxation of this highly phase-correlated state to a prethermalized state, characterized by thermal phase correlations ${ }^{4,19}$. The dynamics is monitored by time-resolved measurements of the relative phase field using matter-wave interferometry ${ }^{20}$.

The experimental procedure starts with a $1 \mathrm{D}$ degenerate gas of $4,000-12,000{ }^{87} \mathrm{Rb}$ atoms trapped at temperatures between $30-110 \mathrm{nK}$ in a magnetic trap, formed $100 \mu \mathrm{m}$ below the trapping wires of an atom chip ${ }^{21}$. By applying radiofrequency fields through additional wires on the chip, we rapidly transform the initial harmonic trapping potential into a double well, thereby realizing the matter-wave analogue of a coherent beamsplitter ${ }^{18}$ (see Methods).

The system is allowed to evolve in the double well for a variable time $t$, before the gases are released by switching off the trapping potential. They expand and interfere after a time-of-flight of $15.7 \mathrm{~ms}$. The resulting interference pattern allows us to extract the relative phase $\phi(z, t)=\theta_{1}(z, t)-\theta_{2}(z, t)$ along the length of the system (Fig. 1). Here, $\theta_{1}(z, t)$ and $\theta_{2}(z, t)$ are the phase profiles of the two individual gases. Repeating this procedure approximately 150 times for each value of $t$, we determine the two-point relative phase correlation function

$$
C\left(\bar{z}=z-z^{\prime}, t\right)=\operatorname{Re}\left\langle\mathrm{e}^{i \phi(z, t)-i \phi\left(z^{\prime}, t\right)}\right\rangle
$$

It measures the degree of correlation between the phases at two arbitrary points $z$ and $z^{\prime}$, separated by a distance $\bar{z}$ (refs 22,23 ). In contrast to the integrated visibility of the interference pattern, which was used in a previous experiment to identify the prethermalized state $^{4}$, the phase correlation function provides a sensitive probe for the local dynamics, and is therefore ideally suited to study the propagation of correlations.

Typical experimental data are presented in Fig. 2a. Directly after the quench, the phase correlation function $C(\bar{z}, t)$ is close to unity for any distance $\bar{z}$. This is a direct manifestation of the long-range phase coherence produced by the splitting process. After a given evolution time $t$, the phase correlation function decays exponentially up to a characteristic distance $\bar{z}_{c}$ and stays nearly constant afterwards: $C\left(\bar{z}>\bar{z}_{c}, t\right)=C\left(\bar{z}_{c}, t\right)$. This means that beyond the distance $\bar{z}_{c}$ long-range phase coherence is retained across the system. With longer evolution time, the position of $\bar{z}_{c}$ shifts to larger distances and the value of $C\left(\bar{z}>\bar{z}_{c}, t\right)$ gradually decreases. This evolution continues until the system reaches a quasi-steady state, where the correlations decay exponentially throughout the entire system $^{19}$. This prethermalized state corresponds to the relaxed state of the 1D system and can be described by a generalized Gibbs ensemble ${ }^{4,10}$. Our observation that the exponentially decreasing parts of the dynamical phase correlation functions match the exponential decay of the relaxed, prethermalized state for $\bar{z}<\bar{z}_{c}$ allows us to conclude that equilibration occurs locally in our system.

From the experimental data, we extract the crossover points $\bar{z}_{c}$ through the level of long-range phase coherence. To this end, we consider for each $t$ the region where the correlation function is constant, extrapolate the constant value to smaller $\bar{z}$ and determine the position $\bar{z}_{c}$ where it crosses the prethermalized correlation function (Supplementary Information). The result of this procedure is shown in Fig. 2b. We observe a clear linear scaling of the position $\bar{z}_{c}=2 c t$, characterizing the local decay of correlations 
a
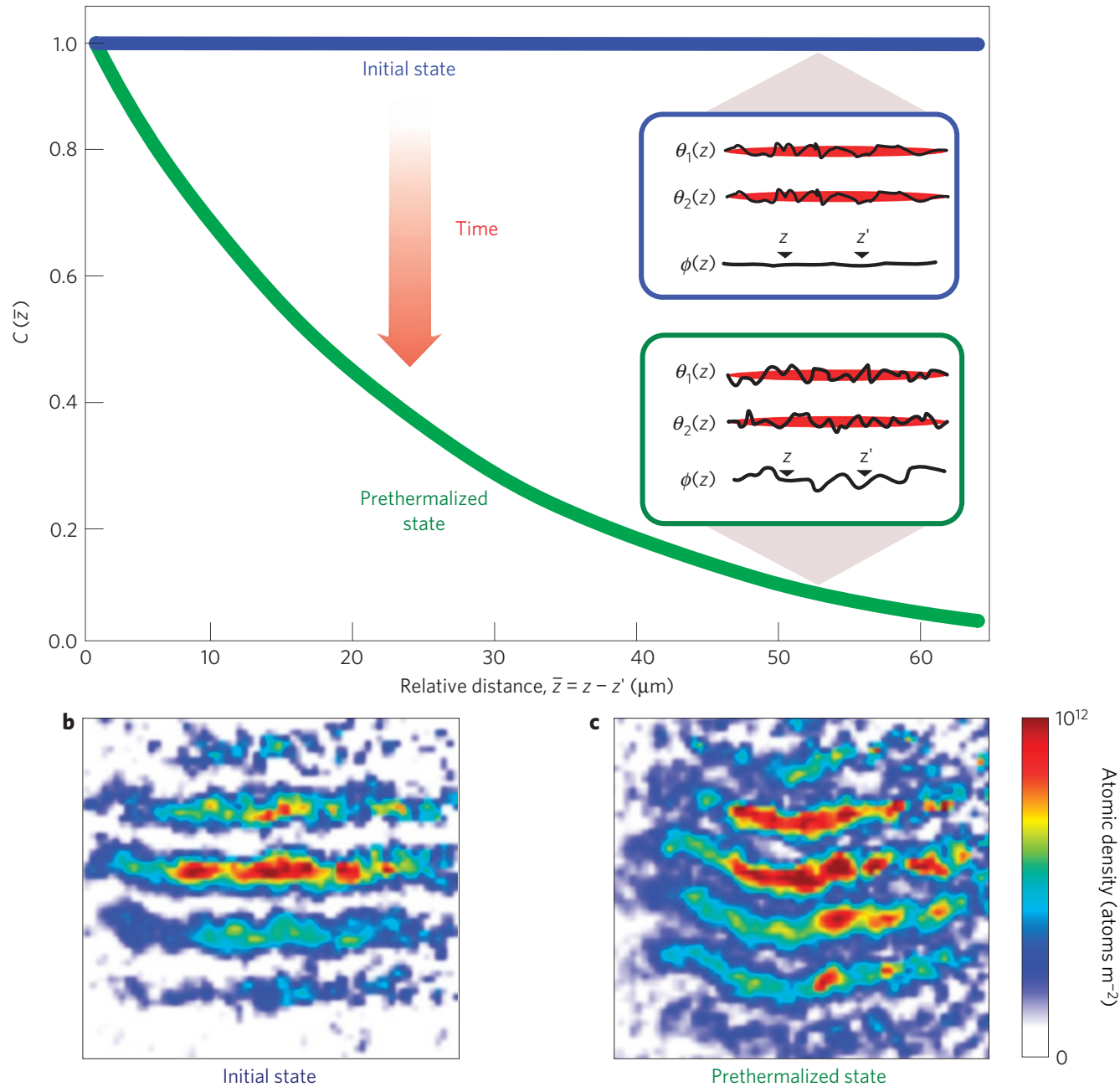

Figure 1 | Characterizing the dynamics of correlations in a coherently split 1D Bose gas. a, The splitting process creates two 1D gases with almost identical longitudinal phase profiles $\theta_{1}(z)$ and $\theta_{2}(z)$, corresponding to long-range phase coherence in the relative phase field $\phi(z)=\theta_{1}(z)-\theta_{2}(z)$. The degree of relative phase correlations between two arbitrary points $z$ and $z^{\prime}$ along the length of the system is characterized by the two-point correlation function $C(\bar{z}, t)$. Initially, it is close to unity for any distance $\bar{z}=z-z^{\prime}$ between the points. Over time, this strongly phase-correlated state relaxes towards a prethermalized state, characterized by thermal (exponentially decaying) correlations. The aim of this study is to investigate how the thermal correlations locally emerge in time. In the experiment, the relative phase field is probed using matter-wave interferometry between the two gases. $\mathbf{b}, \mathbf{c}$, Example interference pictures in the initial (b) and in the prethermalized state (c). In these pictures, the relative phase $\phi(z)$ is directly extracted from the local position of the interference fringes. The phase correlation function is then calculated from an average over approximately 150 interference pictures.

with time. This observation reveals that an arbitrary point in the gas loses its correlations with other points up to a certain separation $\bar{z}_{c}$, whereas long-range phase coherence persists outside this horizon. The experimental data thus show that the prethermalized state locally emerges in a light-cone-like evolution, where $c$ plays the role of a characteristic velocity for the propagation of correlations in the quantum many-body system. For the data presented in Fig. 2b a linear fit allows us to extract a velocity of $c=1.2 \pm 0.1 \mathrm{~mm} \mathrm{~s}^{-1}$.

Light-cone-like effects in quantum many-body dynamics have been previously predicted using results from conformal field theory $^{2}$, and for 2D superfluids ${ }^{24}$. Similarly, it is known that some quantum spin models exhibit an intrinsic maximum velocity ${ }^{6}$ that limits the propagation of correlations and entanglement to an effective light cone $e^{7,25,26}$. It has been conjectured that this leads to a local establishment of thermal properties ${ }^{3}$.

The light-cone-like emergence of thermal correlations that we observe in this work can be understood using a homogeneous Luttinger-liquid model that effectively describes the interacting many-body system in terms of low-energy excitations ${ }^{27}$. Within the Luttinger-liquid model, these excitations are superpositions of phase and density fluctuations. They are characterized by a linear dispersion relation $\omega_{k}=c_{0}|k|$, with $k$ being the momentum of the excitation and $c_{0}$ the speed of sound, the latter defining the characteristic velocity in the homogeneous system.

The coherent splitting process equally distributes energy among the excitations, resulting in a $1 / k$ dependence of their occupation numbers ${ }^{28}$. Each excitation is initialized with small relative phase fluctuations and high relative density fluctuations. Over time, the amplitude of the phase (density) fluctuations increases (decreases), resulting in a progressive randomization of the relative phase field $\phi(z)$. Eventually, the energy associated with the phase fluctuations equilibrates with the energy associated with the density fluctuations, leading to the thermal phase correlations of the prethermalized state ${ }^{28}$.

For a given evolution time $t$, the dephasing of the excitations with different wavelengths $(2 \pi / k)$ randomizes the relative phase field only up to a characteristic distance $\bar{z}_{c}=2 c_{0} t$. This effect can be understood in the following way (see Methods for mathematical details): the degree of randomization of the phase is related to the amplitude of the contributing phase fluctuations. For 

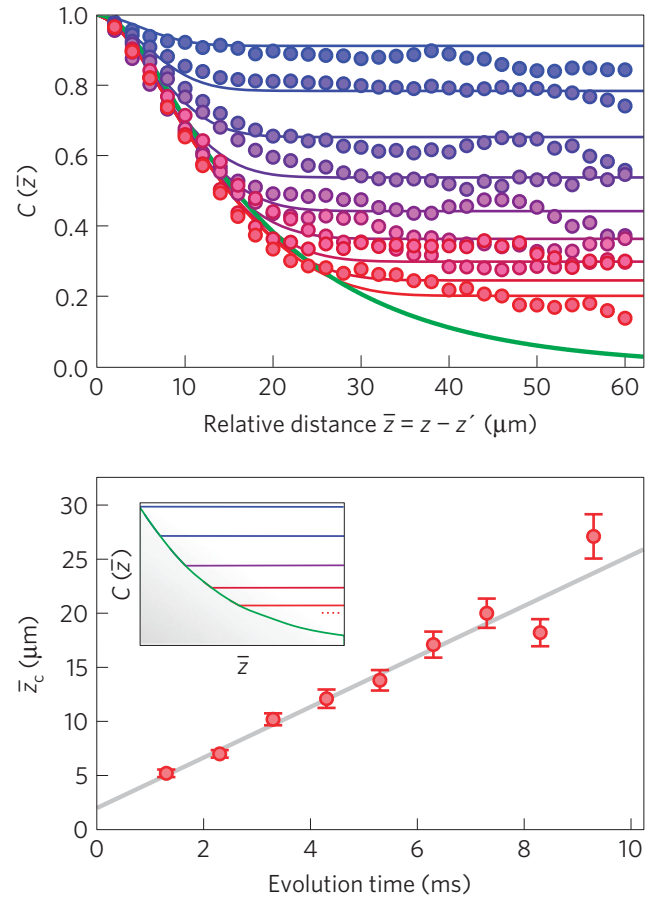

Figure 2 | Local emergence of thermal correlations in a light-cone-like evolution. a, Experimental phase correlation functions $C(\bar{z}, t)$ (filled circles) compared to theoretical calculations (solid lines). From top to bottom, the evolution time $t$ increases from 1 to $9 \mathrm{~ms}$ in steps of $1 \mathrm{~ms}$. The bottom (green) line is the theoretical correlation function of the prethermalized state. For each $t$, the constant values of $C(\bar{z}, t)$ at large $\bar{z}$ can be used to determine the crossover distance $\bar{z}_{c}(t)$ up to which the system forgets the initial long-range phase coherence (see text for details). $\mathbf{b}$, Position of the crossover distance $\bar{z}_{c}$ as a function of evolution time $t$, revealing the light-cone-like decay of correlations. Error bars denote the uncertainty in $\bar{z}_{C}$ following from the standard deviation of the constant values of $C(\bar{z}, t)$ and the uncertainty in the effective temperature of the prethermalized state (see Supplementary Information). The solid line is a linear fit, the slope of which corresponds to twice the characteristic velocity of correlations. Inset: schematic visualization of the dynamics with increasing evolution time from top to bottom as in $\mathbf{a}$. The decay of correlations is characterized by a front moving with a finite velocity: for a given time $t, C(\bar{z}, t)$ is exponential (thermal) only up to the characteristic distance $\bar{z}_{c}(t)$. Beyond this horizon, long-range phase coherence is retained. Note that in the experimental data shown in $\mathbf{a}$, the sharp transitions are smeared out by the finite experimental imaging resolution.

large distances they are associated with the highly occupied long-wavelength excitations that take a long time $\left(\sim 1 / \omega_{k}\right)$ to be converted from the initial density fluctuations into phase fluctuations. At time $t$, there exists a characteristic distance beyond which the contribution of these long-wavelength fluctuations to the randomization of the phase is compensated by a decrease of the contribution from the faster short-wavelength fluctuations (see Supplementary Fig. S3 for an illustration). Therefore, the phase does not randomize any further and long-range phase coherence remains beyond $\bar{z}_{c}$. The sharpness of the transition at $\bar{z}_{c}$ results from the interference of the many excitations with different momenta.

Alternatively, the excitations in the Luttinger-liquid model can also be identified as pairs of quasi-particles, which propagate in opposite directions with momenta $k$ and $-k$, respectively ${ }^{2,7}$. This interpretation naturally leads to the light-cone condition, as two points separated by $\bar{z}$ can establish thermal correlations if quasiparticles originating from these points meet after a time $t=\bar{z} / 2 c_{0}$.

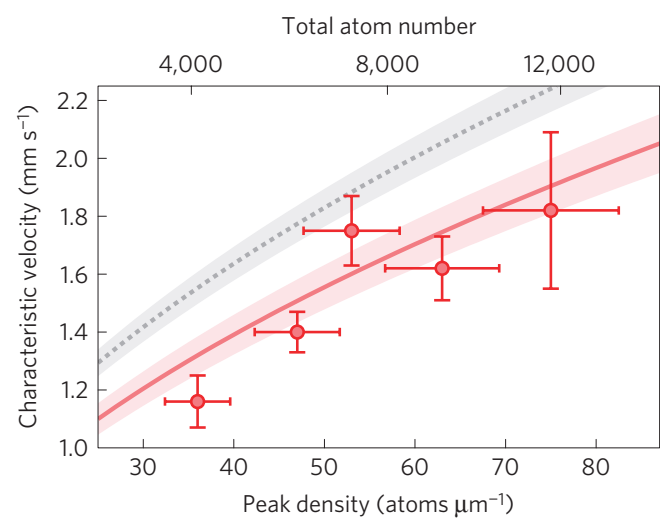

Figure 3 | Scaling of the characteristic velocity with particle number. The solid red (dashed grey) line is the calculated velocity of correlations for a trapped (homogeneous) system. The peak densities are given for each gas. Shaded areas correspond to the uncertainty on the measured trap frequencies. Error bars denote one standard deviation.

In Fig. 2a we compare the results of the Luttinger-liquid calculation to our measured data, taking into account the finite resolution of the imaging system (Supplementary Information). We find good agreement, using independently measured experimental parameters as the input for the theory. This quantitative agreement validates our interpretation of the observations as the local emergence of thermal correlations.

When increasing the number of particles in our quantum many-body system, we expect interaction effects to play a more important role, leading to a faster local relaxation. In the homogeneous limit this is captured by the scaling of the speed of sound $c_{0} \sim \sqrt{\rho}$ with the $1 \mathrm{D}$ density $\rho$ of each gas ${ }^{17}$. To investigate the scaling of the characteristic velocity, we perform the experiment for a varying number of atoms $N$ in the system. We observe the light-cone-like emergence of the thermal correlations over the whole range of probed atom numbers $(N \sim 4,000-12,000)$. In the experimentally realized trapped system, the density varies along the length of the gases, resulting in a spatially dependent speed of sound. Nevertheless, the superposition of many excitations still leads to a single characteristic velocity for the dynamics, which is slightly reduced with respect to the homogeneous case (Supplementary Information). In Fig. 3 we show the measured characteristic velocities. A Luttingerliquid calculation including the trapping potential describes the experimental data within the experimental error, whereas a purely homogeneous calculation clearly overestimates the characteristic velocity.

In our experiment thermal correlations emerge locally in their final prethermalized form. This supports the local relaxation hypothesis ${ }^{3}$ and indicates a general pathway for the emergence of classical properties in isolated quantum many-body systems. In our system, interactions manifest themselves in excitations with a linear dispersion relation (in the homogeneous limit), resulting in a decay of quantum coherence that takes the form of an effective light cone. Whether this scenario holds also for systems with nonlinear dispersion relations, long-range interactions ${ }^{29}$ or systems that are subject to disorder ${ }^{30}$ remains a topic of intense study.

\section{Methods}

Splitting process. The splitting is performed by linearly increasing the amplitude of the radiofrequency current in the chip wires to $24 \mathrm{~mA}$ within $12 \mathrm{~ms}$. To minimize longitudinal excitations during the splitting, the initial gas is prepared in a slightly dressed radiofrequency trap that has the same longitudinal confinement as the final double-well potential (see Supplementary Information for more details). The increase of radiofrequency current results in a rapid decay of the tunnel coupling between the two gases. Simulations of the chip potential and experiments with 
quasi-condensates in thermal equilibrium ${ }^{23}$ indicate that the decoupling of the two gases happens within less than $500 \mu$ s. This is faster than the characteristic timescale of the dynamics ( $\sim 10 \mathrm{~ms}$; ref. 19$)$ and therefore realizes a quench.

Relative phase measurement. The interference patterns are recorded after a time-of-flight expansion of $15.7 \mathrm{~ms}$ using absorption imaging. The point spread function of the optical system has a measured r.m.s. width of $3.6 \mu \mathrm{m}$. The phase $\phi(z)$ of the interference patterns is extracted by fitting each pixel line (of size $\sigma_{\mathrm{px}}=2 \mu \mathrm{m}$ ) with a cosine-modulated Gaussian function.

Theoretical model. Within the Luttinger Liquid theory the phase correlation function can be written as $C\left(z, z^{\prime}, t\right)=\exp \left(-(1 / 2)\left\langle\Delta \phi_{z z^{\prime}}(t)^{2}\right\rangle\right)$, with $\Delta \phi_{z z^{\prime}}(t)=\phi(z, t)-\phi\left(z^{\prime}, t\right)$. In the homogeneous limit, the local relative phase variance is given by ${ }^{28,31}$

$$
\left\langle\Delta \phi_{z z^{\prime}}(t)^{2}\right\rangle=\frac{\pi^{2} \rho}{L K^{2}} \sum_{k \neq 0} \frac{\sin \left(\omega_{k} t\right)^{2}}{k^{2}}(1-\cos (k \bar{z}))
$$

with $L$ being the length of the system, $k=2 \pi n / L$ the momentum of the excitations ( $n \neq 0$ integer) and $K$ the Luttinger parameter. The amount of fluctuations is thus determined by the interference of several longitudinal modes of the $1 \mathrm{D}$ system.

The first term in the sum (1) represents the growth and subsequent oscillations in the amplitude of the phase fluctuations as they get converted from the initial density fluctuations. The factor $1 / k^{2}$ in the amplitude reflects the $1 / k$ scaling of the excitation occupation numbers associated with the equipartition of energy induced by the fast splitting. The second term in the sum corresponds to the spatial fluctuations. Expression (1) is the Fourier decomposition of a trapezoid with a sliding edge at $\bar{z}_{c}=2 c_{0} t$, which explains the two-step feature of the phase correlation function.

A similar expression can be derived for the trapped system probed in the experiment (Supplementary Information).

\section{Received 15 May 2013; accepted 29 July 2013; published online} 8 September 2013

\section{References}

1. Polkovnikov, A., Sengupta, K., Silva, A. \& Vengalattore, M. Nonequilibrium dynamics of closed interacting quantum systems. Rev. Mod. Phys. 83, 863-883 (2011).

2. Calabrese, P. \& Cardy, J. Time dependence of correlation functions following a quantum quench. Phys. Rev. Lett. 96, 011368 (2006).

3. Cramer, M., Dawson, C. M., Eisert, J. \& Osborne, T. J. Exact relaxation in a class of nonequilibrium quantum lattice systems. Phys. Rev. Lett. 100, 030602 (2008).

4. Gring, M. et al. Relaxation and prethermalization in an isolated quantum system. Science 337, 1318-1322 (2012).

5. Berges, J., Borsányi, S. \& Wetterich, C. Prethermalization. Phys. Rev. Lett. 93, 142002 (2004).

6. Lieb, E. H. \& Robinson, D. W. The finite group velocity of quantum spin systems. Commun. Math. Phys. 28, 251-257 (1972).

7. Cheneau, M. et al. Light-cone-like spreading of correlations in a quantum many-body system. Nature 481, 484-487 (2012).

8. Rigol, M., Dunjko, V. \& Olshanii, M. Thermalization and its mechanism for generic isolated quantum systems. Nature 452, 854-858 (2008).

9. Srednicki, M. Chaos and quantum thermalization. Phys. Rev. E 50, 888-901 (1994).

10. Rigol, M., Dunjko, V., Yurovsky, V. \& Olshanii, M. Relaxation in a completely integrable many-body quantum system: An ab initio study of the dynamics of the highly excited states of $1 \mathrm{~d}$ lattice hard-core bosons. Phys. Rev. Lett. 98, 050405 (2007).

11. Kinoshita, T., Wenger, T. \& Weiss, D. A quantum newton's cradle. Nature 440, 900-903 (2006).

12. Gaunt, A. L., Fletcher, R. J., Smith, R. P. \& Hadzibabic, Z. A superheated Bose-condensed gas. Nature Phys. 9, 271-274 (2013).

13. Sadler, L. E., Higbie, J. M., Leslie, S. R., Vengalattore, M. \& Stamper-Kurn, D. M. Spontaneous symmetry breaking in a quenched ferromagnetic spinor Bose-Einstein condensate. Nature 443, 312-315 (2006).
14. Ritter, S. et al. Observing the formation of long-range order during Bose-Einstein condensation. Phys. Rev. Lett. 98, 090402-090402 (2007).

15. Trotzky, S. et al. Probing the relaxation towards equilibrium in an isolated strongly correlated one-dimensional Bose gas. Nature Phys. 8, 325-330 (2012).

16. Gerving, C. S. et al. Non-equilibrium dynamics of an unstable quantum pendulum explored in a spin-1 Bose-Einstein condensate. Nature Commun. 3, 1169 (2012).

17. Petrov, D., Shlyapnikov, G. \& Walraven, J. Regimes of quantum degeneracy in trapped 1d gases. Phys. Rev. Lett. 85, 3745-3749 (2000).

18. Schumm, T. et al. Matter-wave interferometry in a double well on an atom chip. Nature Phys. 1, 57-62 (2005).

19. Kuhnert, M. et al. Multimode dynamics and emergence of a characteristic length scale in a one-dimensional quantum system. Phys. Rev. Lett. 110, 090405 (2013).

20. Cronin, A. D., Schmiedmayer, J. \& Pritchard, D. Optics and interferometry with atoms and molecules. Rev. Mod. Phys. 81, 1051-1129 (2009).

21. Reichel, J. \& Vuletic, V. (eds) Atom Chips (Wiley, 2011).

22. Whitlock, N. K. \& Bouchoule, I. Relative phase fluctuations of two coupled one-dimensional condensates. Phys. Rev. A 68, 053609 (2003).

23. Betz, T. et al. Two-point phase correlations of a one-dimensional bosonic Josephson junction. Phys. Rev. Lett. 106, 020407 (2011).

24. Mathey, L. \& Polkovnikov, A. Light cone dynamics and reverse Kibble-Zurek mechanism in two-dimensional superfluids following a quantum quench. Phys. Rev. A 81, 60033 (2010).

25. Bravyi, S., Hastings, M. B. \& Verstraete, F. Lieb-Robinson bounds and the generation of correlations and topological quantum order. Phys. Rev. Lett. 97, 050401 (2006).

26. Läuchli, A. M. \& Kollath, C. Spreading of correlations and entanglement after a quench in the one-dimensional Bose-Hubbard model. J. Stat. Mech. P05018 (2008).

27. Giamarchi, T. Quantum Physics in One Dimension (Oxford Univ. Press, 2004).

28. Kitagawa, T., Imambekov, A., Schmiedmayer, J. \& Demler, E. The dynamics and prethermalization of one dimensional quantum systems probed through the full distributions of quantum noise. New J. Phys. 13, 073018 (2011).

29. Hauke, P. \& Tagliacozzo, L. Spread of correlations in long-range interacting systems. Preprint at http://arxiv.org/abs/1304.7725 (2013).

30. Burrell, C. K. \& Osborne, T. J. Bounds on the speed of information propagation in disordered quantum spin chains. Phys. Rev. Lett. 99, 167201 (2007).

31. Langen, T. et al. Prethermalization in one-dimensional Bose gases: description by a stochastic Ornstein-Uhlenbeck process. Eur. Phys. J. Special Top. 217, 43-53 (2013).

\section{Acknowledgements}

We would like to thank D. Adu Smith and M. Gring for contributions in the early stage of the experiment, I. Mazets, V. Kasper and J. Berges for discussions and J-F. Schaff and T. Schumm for comments on the manuscript. This work was supported by the Austrian Science Fund (FWF) through the Wittgenstein Prize and the EU through the projects QIBEC and AQUTE. T.L. and M.K. thank the FWF Doctoral Programme CoQuS (W1210); R.G. is supported by the FWF through the Lise Meitner Programme M 1423.

\section{Author contributions}

T.L. and R.G. performed the experiment, analysed the data and carried out the theoretical modelling. J.S. conceived the experiment and the leading scientific questions. All authors contributed to the interpretation of the data and the writing of the manuscript.

\section{Additional information}

Supplementary information is available in the online version of the paper. Reprints and permissions information is available online at www.nature.com/reprints. Correspondence and requests for materials should be addressed to T.L. or J.S.

\section{Competing financial interests}

The authors declare no competing financial interests. 